\title{
Responses to technology and taxes in a simulated world
}

\author{
Detlef P. van Vuuren*, Bert de Vries, Bas Eickhout, Tom Kram \\ National Institute of Public Health and the Environment (RIVM), \\ P.O. Box 1, 3720 BA Bilthoven, The Netherlands
}

Available online 20 July 2004

\begin{abstract}
A set of model experiments was performed to analyze the role of technology development on energy system responses to a global uniform carbon tax. The B2 baseline scenario as implemented by IMAGE 2.2 was used as the starting point for the analysis. Stabilization at a carbon dioxide concentration of 550 ppmv from this baseline was shown to be technically feasible at limited costs. In the first decades, improved energy efficiency and fuel switching play an important role in reducing greenhouse gas emissions. In the longer term, introduction of carbon-free options account for the bulk of the reductions. Technology development is demonstrated to play a crucial role for the mitigation costs (measured as the required level of the imposed carbon tax) by decreasing the gap between the (currently) more expensive low/zero carbon options and their fossil alternatives. For example, technology development, modeled as learning by doing, increases the global carbon reduction in 2030 as a result of a US $\$ 300 / \mathrm{tC}$ tax from nearly $40 \%$ to $60 \%$. Three different aspects of technology development, i.e. technology development under baseline conditions, induced technology development and inertia caused by lifetimes of technologies were identified as important factors in explaining the different responses under different conditions. The relative importance of these factors is of crucial importance for the 'optimal' timing of abatement efforts. Finally, for longterm responses not only has technology development been shown to be important, but also other dynamic processes in the energy system, like depletion. They can sometimes work in the opposite direction.
\end{abstract}

(C) 2004 Elsevier B.V. All rights reserved.

Keywords: Technology development; Energy system; IMAGE 2.2

* Corresponding author. Tel.: +31-30-2742046; fax: +31-30-274-4435.

E-mail address: Detlef.van.vuuren@rivm.nl (D.P. van Vuuren). 


\section{Introduction}

According to the latest report of the IPCC, the climate change observed over the 20th century was mostly caused by human activities (IPCC, 2001b). As further global warming is likely to result in increasing risks of negative impacts on both natural systems and human societies around the world, significant reductions of greenhouse gas emissions may be needed. The IPCC report also indicates that technologies for significantly reducing current emissions with respect to baseline development in the next 20 years are already available. However, reducing emissions on a large enough scale to prevent significant climate change using current technologies is seen in a number of studies to be costly. Therefore development of better technologies needs, certainly in the long run, to play an important role in providing a pathway to further reduce emissions at reasonable costs.

Several tools are used to study pathways to less greenhouse gas intensive futures and the role which might be taken by different (types of) technologies within these pathways (see, for instance, IPCC, 2001a for an overview). In the last few years, the focus on the role of technology development has significantly increased. Several concepts of technology development and its driving forces have been explored, including (descriptions of) autonomous improvement, R\&D-driven improvement and improvement driven by use ('learning-by-doing'). The last category, in particular, has received considerable attention from modelers, both because of its empirical basis and as a way to endogenize technological progress within models (see, for instance, Grubler et al., 1999; Wene, 2000). Understanding the processes that determine technology development, and related to this, the potential of different technological options, is very important for developing mitigation strategies, both in terms of their costs and their timing.

In this paper, we will focus on the role of technology development within different mitigation scenarios and its possible consequences, e.g. for mitigation costs. More specifically, we will search for relevant dynamics within the system that could be important for the role that technological development may play, both in the long and medium terms. Such dynamics include, for instance, the relationship with capital turn-over rates (and inertia in the system), technology development already included in the baseline scenario, development induced by climate policies (both based on learning curves) and the influence of resource depletion. The relative contribution of these different processes is crucial in the debate on the timing of mitigation action.

The analysis was done with the TIMER model, part of the integrated assessment model IMAGE 2.2. The model was developed to study the long-term dynamics of the energy system, in particular, transitions to systems with low carbon emissions (De Vries et al., 2001; IMAGE-team, 2001). TIMER is a system-dynamic energy system model at a medium level of aggregation. The model uses learning curves for almost all its technologies. The position of TIMER within the integrated assessment framework of IMAGE also allows us to study such factors as environmental impacts and co-benefitsbut also land use consequences of mitigation choices. Earlier, the model was used to explore pathways to reach a stabilization of $450 \mathrm{ppmv}$ from the B1 scenario (van Vuuren and de Vries, 2001). In this paper, we will continue this type of analysis by looking at 
different mitigation scenarios that will bring the carbon concentration to $500-600$ ppmv by the end-of-the century, starting from the B2 baseline scenario. ${ }^{1}$

We will first address several methodological issues, including some of the relevant processes of technological change in relation to climate policies, and the most relevant features of the IMAGE/TIMER model. Secondly, we describe how the B2 baseline scenario is implemented in IMAGE/TIMER, providing the context for our further analysis. Thirdly, we will look at the results of the various mitigation experiments explored. These are divided into three experiments. The first investigates how stabilisation of greenhouse gas concentration can be achieved starting from the B2 scenario. The second experiment looks into some of the relevant dynamics of long-term mitigation scenarios (until 2100). The last experiment looks in detail at the different processes relevant for medium-term energy system response to mitigation action. The last section deals with the main conclusions.

\section{Theoretical background and methodology}

The IMAGE 2.2 integrated assessment model and its energy system model TIMER (Alcamo et al., 1998; IMAGE-team, 2001; De Vries et al., 2001), used in the analysis, will be overviewed later in this section. First, we discuss some of the dynamic processes of particular importance for the influence of technology development (assuming the use of learning curves) on the response of the energy system to mitigation action. The modeling experiments are outlined at the end of the section.

\subsection{Dynamic processes that influence technological development}

The main focus here is the role of technology development on the costs of emission reductions in the medium and long term. The term technology development refers to changes in the portfolio of technologies used to supply energy to end-users. In stricter sense it refers to changes to the set of available technologies that change (improve) their performance either in terms of utility or costs. A method to explore the influence of technology development in an energy model is analysis of the response of the model through emission reductions to an externally applied carbon tax. Several authors have used such a method with progressively increasing taxes to develop so-called marginal-abatement cost curves, e.g. Criqui et al. (1999) and Ellerman and Decaux (1998). ${ }^{2}$ We will use this information as a central element of our analysis - defining system response $R$, as indicated in Eq. (1). Here, $E_{\text {tax }}$ represents the emissions after a tax has been applied and $E_{\text {baseline }}$ the emissions in the case of a baseline.

$$
R=E_{\mathrm{tax}} / E_{\text {baseline }}
$$

\footnotetext{
${ }^{1}$ Both the B1 and B2 baseline scenarios are part of total set of six IPCC scenarios introduced in the Special Report on Emission Scenarios (Nakicenovic et al., 2000). B2 is a medium emission scenario in the total set. The scenario will be discussed in more detail later.

2 The curves can be interpreted as marginal-abatement cost curves where the carbon tax is seen as an indicator of mitigation costs. A more general name for these curves is 'system-response' curves.
} 
The focus in this paper is on changes in the system response $R$ as a result of technological change at global level. The use of an energy-system model allows us to study these responses in the context of the (full) dynamics in the world energy system, including depletion and trade but also several technology-relevant processes. In fact, we recognize six dominant dynamic processes in models that are directly related to technological development - and directly influence the response to the model to external impulses. These are:

- switches between different technologies as a result of changes in relative costs;

- the technology development under baseline conditions;

- the induced technology development by applying a carbon tax;

- technology inertia, in particular inertia that due to limited capital turn-over rates;

- investments in research and development;

- impacts of technology-specific resource depletion.

Further on we will discuss these processes in the context of the modeling experiments that have been explored, indicating their importance for the total system response. Here, they are only briefly discussed:

- Switches between different technologies as a result of changes in relative costs. The most direct impact of a carbon tax is that it changes the relative costs of fuels/ technologies and thus also their penetration. This leads to additional use of zero/low carbon fuels/technologies.

- The influence of the technology progress already included in the baseline scenario. In general, costs of new renewable (zero carbon) technologies such as solar/wind and biomass will, under the baseline, decrease more rapidly than the costs of more mature, fossil-based technologies (in a model, this process can be formulated in terms of learning-by-doing or alternatively by exogenous assumptions). As a result, the gap that climate policies need to bridge over time in enforcing the penetration of the more expensive zero carbon options (compared to the cheaper fossil options) decreases. A consequence of this is that, all other things being equal, later introduction of a tax, will meet with a stronger response (in terms of Eq. (1)) than the same tax introduced earlier in time.

- The influence of technology progress induced by climate policies. The learning-bydoing mechanism (see also Section 2.3) implies that further employment of renewable technologies in response to a carbon tax will cause further cost reductions of these technologies. These technologies would then become more attractive, and thus, all other things being equal, the response to a carbon tax would slowly increase over time.

- The influence of technology inertia. There is much inertia in the energy system. Particularly important is that capital is normally only replaced after its lifetime. This means that the response to a carbon tax only slowly precipitates into the system. The response of some energy demand sectors can be somewhat swifter than in other sectors as technical lifetimes of the technologies used are shorter than in energy production and, to some degree, behavioral changes and so-called good housekeeping measures may allow for almost immediate responses. Thus, as a result of inertia, the response to a carbon tax will slowly increase in time. 
- Investment in research and development $(R \& D)$. Another important process that could stimulate technology development is investing in research and development. There is some discussion whether this process can be seen as a separate process for technology development ('learning-by-searching'), or whether it should be seen in conjunction with learning-by-doing. If seen as a separate process, investments into R\&D can bring down costs of more expensive low carbon options without applying these technologies first, increasing the response to a carbon tax in time.

- The influence of depletion. Indirectly, the use of a carbon tax also changes the depletion dynamics of different forms of energy. Important in this context is that different fuels/ technologies have their own depletion characteristics.

It is interesting to see that these different processes are strongly related to the earlier discussion on the timing of mitigation action. The second process (learning in the baseline) leads to the conclusion that it is better to wait for technologies to develop before implementing strict climate policies. This argument was forcefully presented in (Wigley et al., 1996) in their discussion on timing of mitigation action. In contrast, the third process enforces the argument that climate policies should be seen as a lever with which to bring about climate-friendly technical innovation and diffusion, favoring an early-action type of approach (Wene, 2000; Azar and Dowlatabadi, 1999; van Vuuren and de Vries, 2001). The fourth process translates into an argument that no climate policy should result in premature replacement of capital. This argument was used by Wigley et al. (1996) as a reason for later abatement being cheaper. However, others have argued that after including fully all system inertia, this argument actually gives preference to early action to make the transition as smooth as possible (Ha-Duong et al., 1997; Grubb, 1997). The fifth process might, in turn, favor a strategy in which first strong investments into R\&D are made, followed latter by large-scale employment of available technologies (once they have become competitive). Finally, the influence of the sixth process is ambiguous. A crucial question from a final decision on timing is how important these processes are in relation to each other.

In an earlier publication, we looked into how the total set of processes could work out in a scenario with very positive assumptions on technology development and low energy use (the SRES B1 scenario) (van Vuuren and de Vries, 2001). We found early action to be a more favorable strategy than delayed response for a discount rate of $4 \%$ and lower, as postponing measures foregoes the benefits of learning-by-doing. Using higher discount rates would favor a delayed response approach.

\subsection{Modeling framework}

In this study we used the TIMER energy system model and the integrated assessment framework IMAGE 2.2. ${ }^{3}$ IMAGE 2.2 was developed to assess the impact of global environmental problems, in particular, climate change. It actually consists of a set of linked and integrated models collectively describing the chain of global environmental change from population and economic change to impacts on ecosystems and agricultural systems.

\footnotetext{
3 An abbreviation of Integrated Model to Assess the Global Environment.
} 
The models operate on two geographical scales. Most of the drivers of change (population, economy, agricultural demand, energy use, emissions) are calculated for 17 world regions. In addition, a large number of the environmental parameters are calculated at the grid level of $0.5^{\circ} \times 0.5^{\circ}$. The IMAGE 2.2 scenarios cover the 1970-2100 period. In 2001, the model was used to re-implement the IPCC SRES scenarios (base year updated to 1995) (see IMAGE-team, 2001).

\subsubsection{TIMER}

TIMER is an energy-system model describing the demand and supply of 12 different energy carriers for 17 world regions (Fig. 1). A full description of the model can be found in (De Vries et al., 2001). The main objective of the TIMER model is to analyze the long-term trends in energy demand and efficiency and the possible transition towards renewable sources. The model particularly focuses on several dynamic relationships within the energy system, such as inertia, learning-by-doing, depletion and trade among the different regions. This makes the model very suitable to study some of the long-term dynamics related to technology development discussed in Section 2.1.

The demand submodel of TIMER determines demand for fuels and electricity in five sectors (industry, transport, residential, services and other) based on structural change, autonomous and price-induced change in energy intensity ('energy conservation') and price-based fuel substitution. The demand for electricity is fulfilled by fossil-fuel based thermal power, hydropower and two other non-thermal alternatives, i.e. nuclear power and

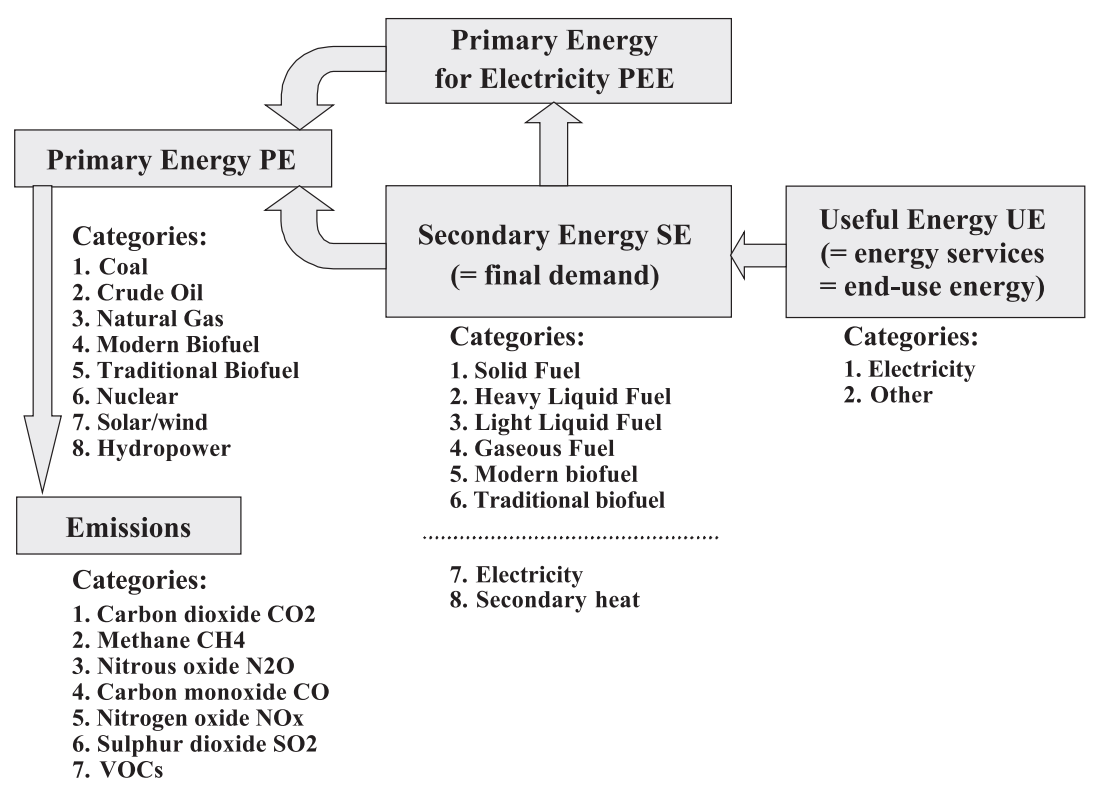

Fig. 1. Overview of the TIMER model. 
solar/wind. The option 'solar/wind' describes a renewable electricity option with characteristics of both solar and wind power. Both nuclear power and solar/wind penetrate the market based on relative costs. The thermal power option consists of four alternative options: coal based, oil based, natural-gas based and biomass based which are fully intercompetitive. The exploration and exploitation of fossil fuels (either for electricity or direct fuel use) is described in terms of depletion and technological development. Biofuels can be used in place of fossil fuels, and are on their turn also assumed to be subject to technological development and depletion dynamics.

Below we will describe those processes in TIMER that directly relate to the dynamic processes discussed in Section 2.1.

\subsubsection{Technology development}

An important aspect of the TIMER model is the endogenous formulation of technological development on the basis of 'learning-by-doing'. This phenomenon has been investigated in detail and for a variety of products and processes. More recently, the concept received great interest as a meaningful representation of technological change in global energy models (Grubler et al., 1999; Azar and Dowlatabadi, 1999; Wene, 2000). Its general formulation is that a cost measure $y$ tends to decline as a power function of an accumulated learning measure, $x$ :

$$
y=\alpha^{*} Q^{-\pi}
$$

where $\pi$ is the learning rate, $Q$ the cumulative output and $\alpha$ a constant. Often, the learning rate, $\pi$, is expressed by the progress ratio $\rho$, which indicates the factor with which the costs measure, $y$, decreases with the doubling of experience, $x$. It is easily seen that $\rho=2^{-\pi}$. Many illustrations of this law have been found and published. The progress ratio in almost all cases investigated has been found to be between 0.65 and 0.95 , with a median value of 0.82 (e.g. Argotte and Epple, 1990).

In the TIMER model, the learning factor influences the costs of coal, oil and gas production, the investments of renewable and nuclear energy and the decline of the energy conservation cost curves. The value of the learning rate $\rho$ varies from a high of 0.7 to 1.0 based on historic $\rho$ values for the different technologies. The choice of these values depends on the technologies and scenario setting. First of all, the learning rates of solar/ wind and biomass have been set lower than those for fossil-based technologies, based on observed historic trends (e.g. Wene, 2000). There is strong evidence that in the early stages of development $\rho$ values for learning-by-doing curves are lower (thus faster learning) than for technologies that have already been in use for long-time periods. Some have tried to explain this by other factors, which may contribute to learning in these early phases, such as a relatively high investment in research and development (Grubler et al., 1999; Wene, 2000). In TIMER all $\rho$ values are time-dependent with $\rho$ values going to 0.9 or higher before 2100 for all technologies. The development of the learning rates is also related to the storyline of the scenario (e.g. high technological progress or not). Table 1 gives the $\rho$ values used in the B2 scenario of TIMER.

An interesting question is whether learning curves should be applied at the level of regions or for the world as a whole. On the one hand, technologies developed in one 
Table 1

Progress ratios used in the B2 scenario as implemented in TIMER

\begin{tabular}{lll}
\hline Technology & Progress ratio 1995 & Progress ratio 2100 \\
\hline Coal production & $0.90-0.94$ & $0.95-0.96$ \\
Oil production & 0.85 & 0.92 \\
Natural gas production & $0.86-0.93$ & 0.90 \\
Efficiency & $0.85-0.9$ & 0.92 \\
Nuclear & 1.00 & 0.96 \\
Solar/wind & 0.80 & 0.90 \\
Biomass & 0.88 & 0.92 \\
\hline
\end{tabular}

region will, in most cases, also be available in other regions. On the other hand, a significant part of cost reductions is actually representative of the experience gained by applying the technology. In TIMER, the learning curves are applied at the level of separate regions; however, to model the influence of technology transfer, we assume that all other regions will partly benefit from the additional knowledge gain of the forerunner (see Vries et al., 2001).

\subsubsection{Depletion}

Depletion plays a different role for different technologies. Depletion is described in terms of long-term supply curves (related to cumulative production) for the fossil fuel technologies and nuclear energy. These curves are derived from (Rogner, 1997) and the World Energy Assessment (WEA, 2000). For renewable sources, in contrast, depletion is described as a function of production. This formulation assumes that at higher production levels less attractive sites or technologies will have to be used. Specific investment costs and the maximum production levels for renewable energy have been derived from various sources, as indicated in the model documentation (De Vries et al., 2001). These derived values include, in particular, the resource estimates of the World Energy Assessment and calculations made using the IMAGE 2.2 land use model (WEA, 2000, Hoogwijk et al., 2000). A specific form of 'depletion' is found within the electricity sector-where it is assumed that only a limited share of renewable electricity options can be adopted free of costs - after which additional investments need to be made into the system to assure sufficient reliability (e.g. storage or grid extensions). These additional costs are assumed to come into play where the share of solar/wind in total electricity production is above $20 \%$.

\subsubsection{Substitution between different technologies}

Substitution among energy carriers and technologies is described in the model with the multinomial logit model (Edmonds and Reilly, 1985):

$$
\mathrm{IMS}_{i}=\exp \left(-\lambda^{*} c_{i}\right) / \sum_{j} \exp \left(-\lambda^{*} c_{j}\right)
$$

$\mathrm{IMS}_{i}$ is the indicated share in total investments of production method $i, \lambda$ the so-called logit parameter determining the sensitivity of markets to price changes and $c_{i}$ the costs or 
the price of production method $i$. The latter may include other factors such as premium factors, additional investment costs and cost increases as result of a carbon tax. The multinomial logit model used here indicates that the share of a certain technology (or fuel type) depends on its relative costs to its competitors. The cheapest option gains the largest market share. However, it does not get the full market share as we assume heterogeneity within the market, creating specific niches for technologies with higher average costs (but lower costs than its alternatives within this specific niche). The multinomial logit mechanism is used within TIMER to describe substitution among end-use energy carriers, different forms of electricity generation (coal, oil, natural gas, solar/wind and nuclear) and substitution between fossil fuels and biofuels. It should be noted that the mechanism is actually used to determine shares in new investment, which implies that actual market shares respond much slower.

\subsection{Modeling experiments}

In order to learn more about the possible role of different technology pathways, we performed three different model experiments, starting from IMAGE implementation of the SRES B2 scenario, i.e.:

(a) A scenario aimed at stabilization of atmospheric carbon dioxide concentration at 550 ppmv;

(b) A series of three model runs in which a US\$100/tC carbon tax is introduced: (i) immediately going from zero to US $\$ 100 / \mathrm{tC}$ between 2000 and 2010; (ii) increasing at US $\$ 25 / \mathrm{tC}$ per decade in the first 40 years after 2000 - and staying constant at US\$100/ tC after 2040 and (iii) increasing at US\$10/tC per decade for the whole 2000-2100 period (see Fig. 5);

(c) A series of model runs in which different levels of carbon taxes are applied in 2000, 2010, 2020 and 2030, with the response recorded 10-30 years afterwards.

In the first experiment, we looked at the types of technologies chosen by the model to achieve the required level of mitigation. Some attention was paid to possible consequences of this action, for instance, in terms of reduction of other greenhouse gasses and impacts on energy-exporting regions. In the second set of experiments, a carbon tax was introduced in three different modeling runs, in all cases reaching a level of US\$100/tC (see Fig. 5), but the rate of introduction varied between the different experiments. The aim of this experiment was to find out whether technology dynamics within the system would result in different responses to these taxes in the long term. Specifically, one may expect that the run reaching the final US\$100/tC tax level early in the simulation would benefit more from the induced technology development than any of the other runs. The last set of experiments took place in a much shorter time frame and searched specifically for the different contributions to the overall system in its response to a carbon tax of induced technological learning where learning forms part of the baseline and inertia.

It is important to note that the model applied in this study does not take into account physical carbon sequestration (removing carbon from the energy system for underground/ underwater storage) or options to reduce land-use related emissions. 


\section{Baseline scenario}

The IPCC SRES B2 scenario has been developed within a total set of six baseline scenarios, none of which includes explicit climate policies (Nakicenovic et al., 2000). The IPCC SRES scenarios are based on the development of narrative 'storylines' and the quantification of these storylines using six different integrated models from different countries. For each scenario, the elaboration by one specific model has been chosen as being characteristic for that particular storyline, the so-called marker scenario. Elaboration of the same storyline by other models needs to fulfil certain harmonization criteria before they can be indicated as a fully harmonized elaboration. The B2 storyline describes a regionalised world with a focus on environmental and social values. The marker implementation of the B2 scenario strongly emphasizes the 'dynamics-as-usual' results that could play a role in this scenario (Riahi and Roehrl, 2000). The IMAGE 2.2 implementation, in contrast, has put slightly more emphasis on the original storylinealthough the implementation still fully complies with the harmonization criteria (IMAGE-team, 2001). As a result, the IMAGE B2 scenario has slightly lower emissions than the original marker but can still be regarded as a medium emission scenario with global greenhouse gas emissions increasing from $10 \mathrm{GtC}$-eq in 2000 to about $15 \mathrm{GtC}$ eq in 2020 - and finally stabilizing at 17 GtC-eq from 2040 onwards (see Fig. 2). In terms of sectors, energy use remains responsible for the larger share of emissions. Driven by the increasing emissions, the atmospheric carbon dioxide concentration in the

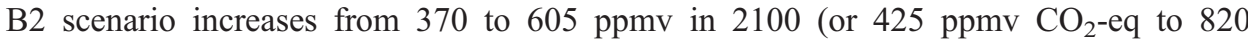
ppmv $\mathrm{CO}_{2}$-eq), more than double pre-industrial levels. The global temperature increase is found in the range of almost $3^{\circ}$ above 1970 levels (using a central estimate of climate sensitivity).

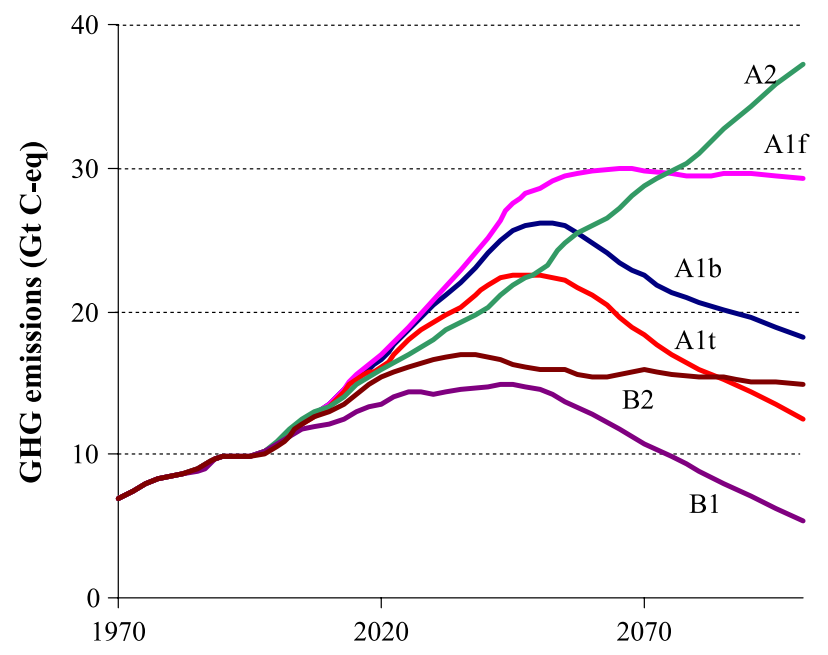

Fig. 2. Global greenhouse gas emissions in the IMAGE 2.2 implementation of the SRES scenarios (all Kyoto gases and all sources) (IMAGE-team, 2001). 


\section{Mitigation experiments}

The results of the experiments described in Section 2.3 are outlined below.

\subsection{Stabilization at 550 ppmv}

Reaching a profile that stabilizes the atmospheric carbon dioxide concentration at 550 ppmv from the IMAGE B2 scenario requires a reduction of cumulative emissions in the $2000-2100$ period of about $25 \%$. Such reduction could be regarded as a relatively modest one. ${ }^{4}$ If we introduce a uniform carbon tax (across regions and sectors) in TIMER, we need a tax slowly rising to US\$190/tC to achieve such a reduction (no carbon tax is applied to land-use-related carbon emissions). The profile of the required carbon tax is shown in Fig. 5.

In total, the required carbon tax reduces global primary energy use by about $10-15 \%$. This decrease is unequally divided among the different energy carriers. Cumulative use of coal declines by almost 50\%. The cumulative consumption of natural gas and oil declines by about $10 \%$, respectively (the decline in natural gas is slightly higher than for oil, as natural gas experiences considerable competition from non-fossil energy carriers in the electricity market). Other, low/zero carbon, energy carriers gain market share such as modern biomass $(+14 \%)$ and nuclear and renewable power electricity (in total $36 \%$ gain).

In Fig. 3, we attributed the reduction in carbon emissions from B2 to B2-550 to the different changes within the system. ${ }^{5}$ In the first two decades, the lion's share of the reductions comes from energy efficiency improvement and fuel-switch from coal to other fossil fuels. By 2030, the other options start to become important: using biofuels instead of fossil fuels and non-thermal electricity modes (solar/wind and nuclear power) instead of fossil-based electricity. ${ }^{6}$ The largest reductions are likely to occur in the electrical power sector. This result can easily be understood if one looks at generation costs of the two fully competitive non-fossil power options compared to those of thermal power (Fig. 4). In the baseline, from 2000 until around 2030 there is still a very clear gap between the generation costs of these options in favor of fossil-fuel based options; solar/wind is still around a factor 2-3 times more expensive, while the difference with nuclear power is somewhat smaller. In time, by learning-by-doing, the costs of solar/wind power and nuclear power slowly decline and around 2050 generation costs become nearly equal. As solar/wind power at that time gain a considerable market share, cost reductions start to be offset by the

\footnotetext{
4 The reduction is in size of the same magnitude as the reduction that is required for achieving stabilisation at $450 \mathrm{ppmv}$ of carbon dioxide in the atmosphere starting from the B1 baseline scenario that we described earlier (van Vuuren and de Vries, 2001). Further in this section we compare the results to those of the B1 $450 \mathrm{ppmv}$ analysis.

5 The actual size of each option depends somewhat on the order of attribution. We first determined the total contribution from efficiency improvement, next from penetration of solar/wind and nuclear power and biofuels, then from biofuel penetration and finally for a fuel-switch among the different fossil fuels.

6 We have allowed additional use of nuclear power as a mitigation option in these calculations. In fact, as the cost of this option is lower in the baseline than the solar/wind power option, it represents the most attractive alternative in terms of a first response. The 'learning' capacity of this option is, however, assumed to be lower than for solar/wind power. It should be noted that generation costs for fossil-based electricity is in fact calculated in the model through a weighted average of coal, oil and natural gas generation costs.
} 


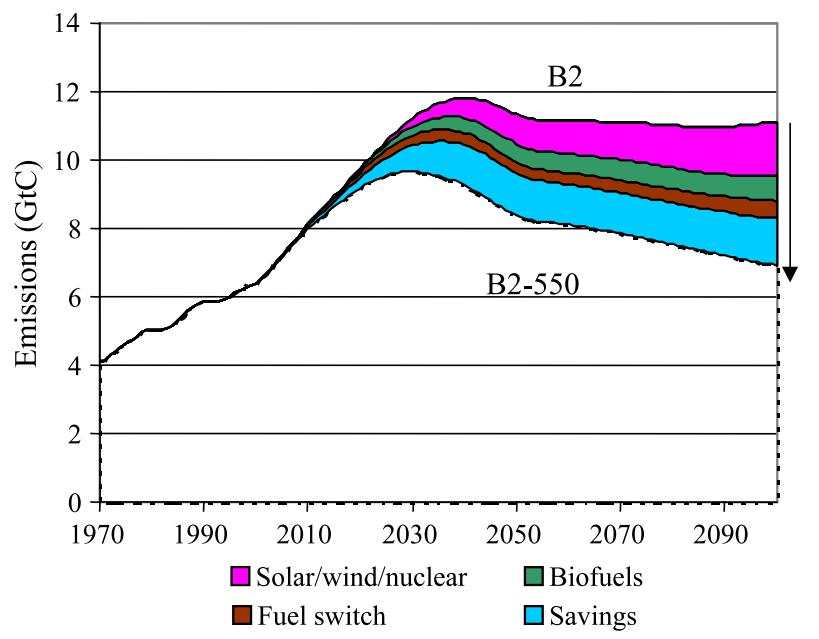

Fig. 3. Allocation of carbon dioxide emission reduction going from B2 to a $550 \mathrm{ppmv}$ stabilization scenario.

fact that the best production sites are already occupied and the further penetration requires higher storage and/or distribution costs. As a result thermal-based electricity remains the cheapest of the supply options throughout the century in the baseline. If a carbon tax is introduced into this system, it easily shifts the costs of the thermal options above the alternative costs for nuclear and solar/wind. This induces in the model a strong penetration of these options into the power generation system, allowing for a sharp reduction of carbon dioxide emissions.

The strongest impact of the carbon tax is on coal use. Hence, the largest changes in terms of energy use will occur in regions with relatively high coal consumption and production rates. This includes China, India, South Africa and the USA. Impacts on oil use and trade are much smaller - in view of the relatively modest taxes required to reach 550 from the B2 scenario (also note that trade levels in B2 are somewhat lower than in other SRES scenarios). Middle East oil exports, for instance, decrease in terms of the ratio of export revenues and GDP from $11.6 \%$ to $11.1 \%$ in the $2000-2050$ period (Table 2).

Table 2

Volume of fuel trade as \% of GDP in selected regions (2000-2050) (net imports are negative; net exports are positive)

\begin{tabular}{|c|c|c|c|c|c|c|}
\hline & \multicolumn{3}{|c|}{ Oil export (\% GDP) } & \multicolumn{3}{|c|}{ All energy export (\% GDP) } \\
\hline & B2 & B2-550 & Diff. & B2 & B2-550 & Diff. \\
\hline USA & -0.75 & -0.71 & 0.04 & -1.36 & -1.41 & -0.04 \\
\hline South America & 1.09 & 1.02 & -0.07 & 2.44 & 2.70 & 0.26 \\
\hline Western Europe & -0.57 & -0.52 & 0.05 & -1.14 & -1.12 & 0.02 \\
\hline FSU & 3.37 & 3.01 & -0.36 & 10.44 & 10.97 & 0.53 \\
\hline Middle East & 11.64 & 11.13 & -0.51 & 13.58 & 13.11 & -0.47 \\
\hline South Asia & -2.07 & -1.93 & 0.14 & -3.54 & -3.53 & 0.00 \\
\hline East Asia & -0.70 & 0.67 & 0.03 & -1.11 & -1.22 & -0.11 \\
\hline Japan & -0.66 & -0.63 & 0.03 & -1.28 & -1.25 & 0.03 \\
\hline
\end{tabular}



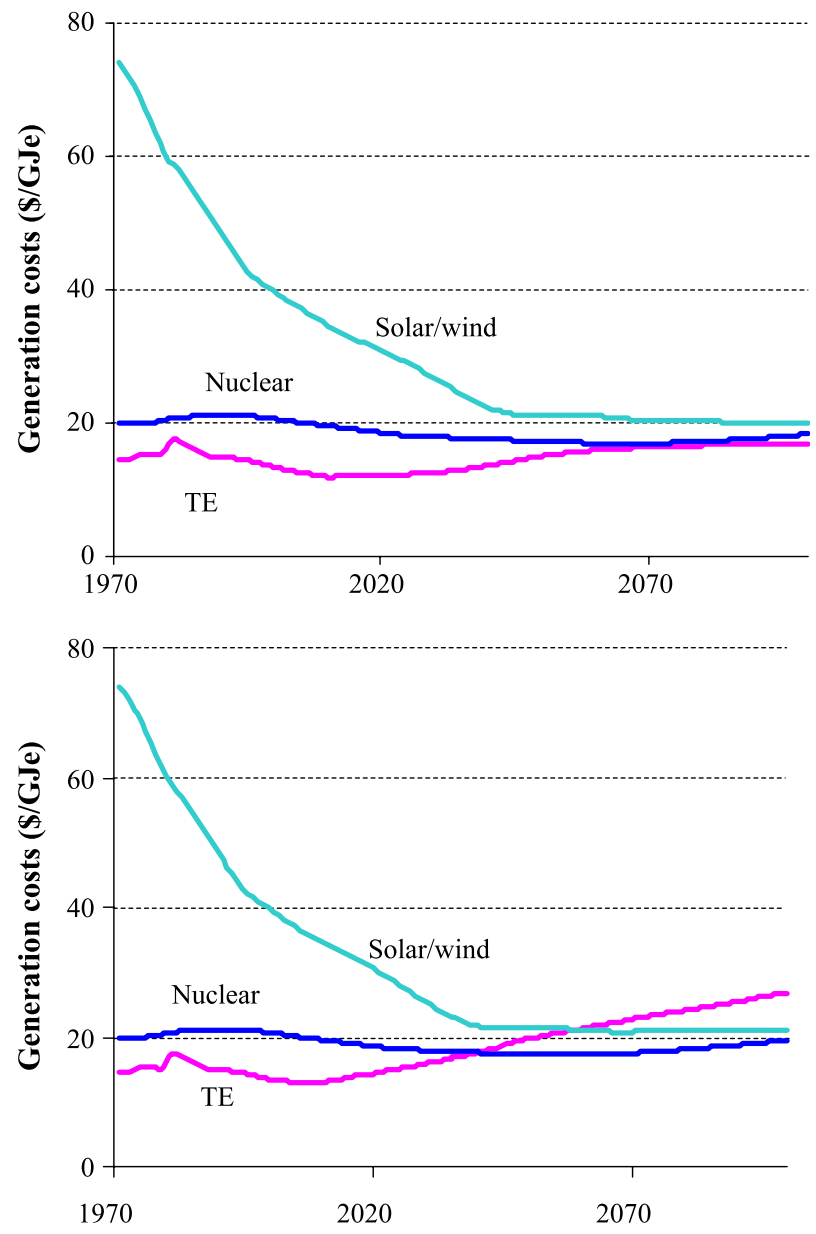

Fig. 4. Generation costs of non-thermal options (solar/wind and nuclear) versus electricity from thermal-power plants (mostly fossil-fueled, but including biofuels; TE) electricity generation in the B2 baseline (top) and the 550 stabilization scenario (bottom).

Impacts in regions with slightly higher production costs, such as the FSU, could be larger in relative terms. A number of other import regions could benefit from reduced oil imports, around 2050 in particular China and India. Interestingly, changes in the trade of other fuels can cause a different picture for total energy export as percentage of GDP. The Former Soviet Union, for instance, is suffering in the long-run (2030-2060) from reduced oil exports. The exploitation from this region's oil resources, very competitive by that time under the baseline, is subject to a carbon tax that by that time already reaches a level of US $\$ 100 / t C$. In contrast, in the medium term (2010-2030) this region benefits significantly from increased natural gas exports to Western Europe and Japan. Also, South America sees some losses in oil exports - but these are offset as the region captures its experience in producing biofuels and becoming an important exporter of these fuels. Finally, for China, 
the reduction in oil exports is offset by an equally sharp increase in natural gas and later biofuels imports (van Vuuren et al., 2003).

The reduction of energy/industry-related cumulative carbon dioxide emissions are about $20 \%$, following a $20 \%$ in 2050 and a $40 \%$ in 2100 (the equivalent of $4.3 \mathrm{GtC} /$ year). As a result of the induced changes in the energy system to the carbon tax (more energy crops to produce biofuels, thus less land for new forest), land-use emissions increase slightly by about $0.4 \mathrm{GtC}$ (a form of carbon leakage that could be reduced by additional policies oriented to land-use related emissions). The carbon tax does not directly tax non-carbon dioxide greenhouse gasses either. However, as the carbon tax induces changes in the energy system, the emissions of other energy-related gasses are reduced. For instance, energy-related methane emissions are reduced by about $10 \%$ compared to baseline (a $60 \%$ increase of emissions instead of a $70 \%$ increase) - with corresponding advantages in terms of greenhouse gas concentrations. Sulfur emissions are also reduced by about $10 \%$ compared to baseline. The latter gives rise to important co-benefits of climate policies in terms of reduction of both urban and regional air pollution (van Vuuren et al., 2003).

The B2-550 stabilization scenario developed here results in a rise of global average temperature of $2.6^{\circ} \mathrm{C}$ vis-à-vis a temperature increase of $2.9^{\circ} \mathrm{C}$ in the $\mathrm{B} 2$ baseline scenario. The gains from reduction of the radiative forcing of carbon dioxide are, in particular in the first decades, somewhat offset by a decrease in the negative forcing of sulfur aerosols.

If we compare the results for stabilizing the carbon concentration at $550 \mathrm{ppmv}$ from the B2 scenario to our earlier analysis, we see that the required efforts and consequences are very comparable. Stabilizing the carbon concentration at $450 \mathrm{ppmv}$ from the B1 scenario required a US\$200-230/tC carbon tax by the end of the century (depending on the timing), versus the US\$190/tC used here. Responses in terms of the contribution of different technologies also seems to be comparable — although reducing coal use is slightly more important in this B2-550 analysis in view of the higher shares of coal use in total energy use. In contrast, impacts on the oil trade are smaller-most probably due to the more fragmented oil market in the B2 scenario.

\subsection{Responses to different US\$100/tC taxes}

In the second set of experiments, a carbon tax is introduced that reaches a level of US $\$ 100 / \mathrm{tC}$ - but is introduced at three different rates (see Fig. 5). Fig. 6 shows that carbon dioxide emissions are reduced the fastest in the scenario that has already reached the US\$100 level in 2010 (1); followed by the second and third scenario. As a result, by 2100 the first scenario has a considerably lower carbon dioxide concentration than the third. We can also compare the relative reductions for the same tax levels. These are not always similar; apparently, model dynamics do play a role here. However, the expected effect (see Section 2) of a sharper 2100 emission reduction in the first scenario compared to the others, due to a longer period of induced learning, is not visible. There are four important reasons inherent in the model for this:

- Learning slows down with knowledge gained. The learning curve describes technical progress as a function of the logarithm of cumulative production. This means that a 


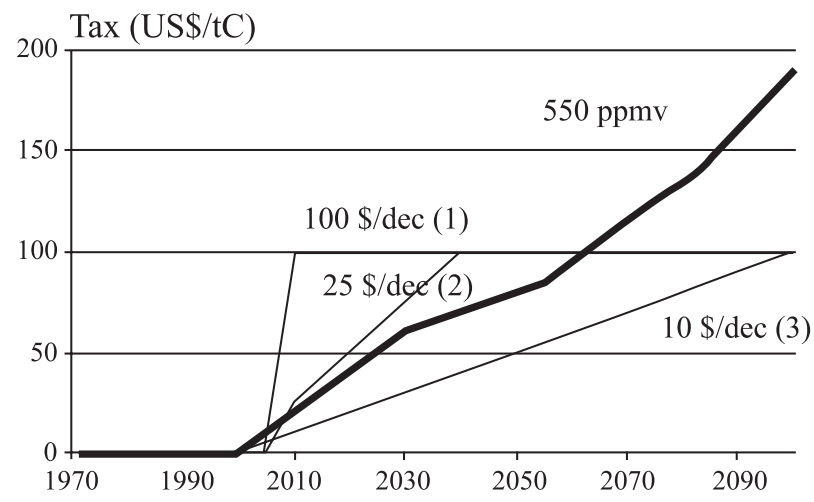

Fig. 5. Overview of the different taxes applied.

similar improvement in production costs can be realized for each doubling of cumulative production, as explained in Section 2. Production itself cannot keep 'doubling' its production rates throughout the century, thus cost reductions slow down in time. The scenario that reaches the US\$100/tC as early as 2010 benefits from fast learning early in the scenario-but also experiences the consequences of slower learning afterwards.

- High production rates for renewables are costly. We assume that depletion for renewable technology option is directly related to production rates (see Section 2): high production rates imply that less favorable options (e.g. less favorable sites for wind power) have to be chosen. The early tax scenario results in higher production rates of these options - and thus experiences higher depletion.

- High shares of renewables induce costs. Most of the renewable energy options have lower reliability in terms of their electricity production than fossil-fuel options. Therefore, total electricity production can only take up a certain percentage of renewable electricity options (we assumed 20\%) before requiring additional investments into the system to improve its reliability (e.g. storage or grid extensions that enlarge the system). This dynamic element has similar results to the one for depletion described above.

- Some cheap oil and gas are still there. Finally, the competitive fossil-based alternatives will have slightly lower production costs in the first scenario than in the second and third scenario as less depletion of cheap resources will have taken place.

In conclusion, in addition to 'learning-by-doing' there are also other technologyrelevant dynamic processes, some of which may work in the opposite direction to the expected gains for early action scenarios of 'learning-by-doing'. Under the B2 model assumptions in TIMER, these processes completely offset the gains of early action in terms of costs by 2100 . On the other hand, it should be noted that the early action benefited from lower costs for solar/wind during most of the simulation (see Fig. 7). The environmental impacts of these three scenarios are certainly not similar (see carbon dioxide concentration in Fig. 6). 

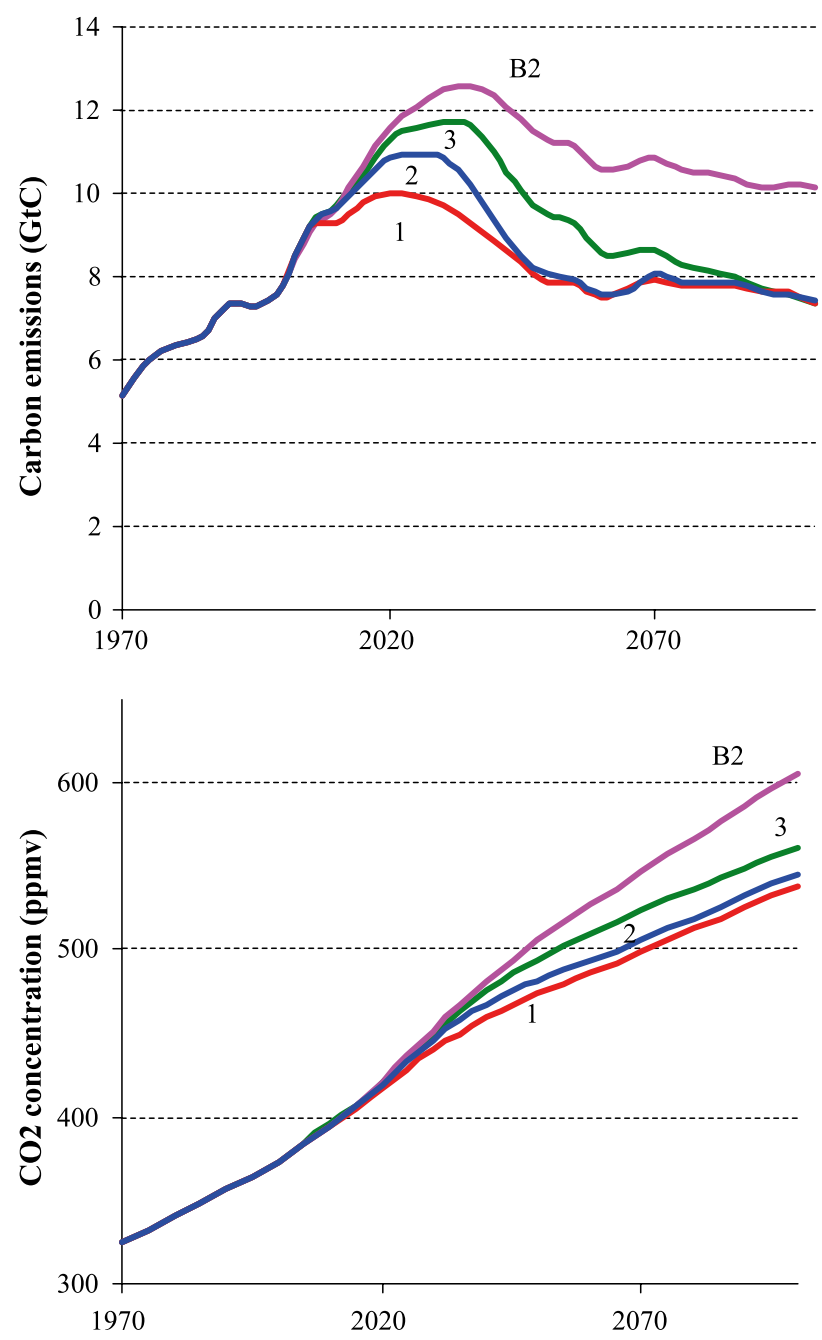

Fig. 6. Global carbon dioxide emissions (top) and carbon dioxide concentration (bottom). Note: the numbers correspond to the different tax profiles of Fig. 5.

\subsection{Responses to carbon tax with and without learning}

In the last set of experiments, we took a shorter time horizon (2000-2030) and investigated whether we could identify the role of different relevant dynamics to determine response to a carbon tax as defined in formula (1). We assumed that some of the dynamics discussed in the previous section were of less importance on this medium-term time scale, in particular those related to depletion. The three types of dynamics of particular importance for the medium-term response are technology development under baseline, induced technology development and system inertia. 


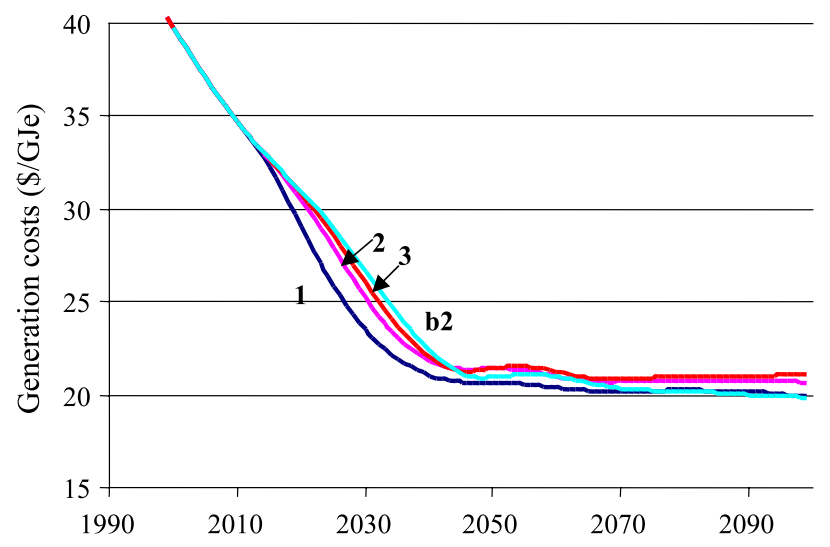

Fig. 7. Costs of solar/wind power generation in TIMER. Note: the numbers correspond to the different tax profiles of Fig. 5.

We tried to get an idea of the influence of the three processes through a set of experiments in which we recorded the system response as a function of the year of introduction $\left(t_{\text {in }}\right)$, the year in we measure the system response $\left(t_{\text {rec }}\right)$ and the level of the tax $(T)$. For both $t_{\text {in }}$ and $t_{\text {rec }}$, values were applied in 5-year steps between 2000 and 2030. The level of the carbon tax varied between US\$0 and 600/tC.

In the first experiment we focused on the recording year $\left(t_{\text {rec }}\right)$. We introduced a carbon tax into the TIMER model in the year $2000\left(t_{\text {in }}\right)$ of US\$10/tC $(T)$ and recorded its immediate impact in 2000, and its impact in 2010, 2020 and $2030\left(t_{\text {rec }}\right)$, after 10, 20 and 30 years, respectively. This experiment was repeated for the different tax levels between US $\$ 10$ and $600 / \mathrm{tC}$ in steps of US\$10/tC. This process is very similar to experiments in which modelers record the response of their model to carbon taxes in order to derive socalled Marginal Abatement Curves (MAC). However, in contrast to the normal MAC experiments, we looked at how the system response develops over time in the period after introduction of the carbon tax. Fig. 8a shows the results of this experiment. The recordings have resulted in four system-response curves that indicate the reduction in global carbon emissions in four different years. All of the curves show the typical form of a MAC, in which the response increases along with the level of the tax but with decreasing additional gains. Fig. 8a shows the response to the carbon tax increasing with time. A US\$300 tax introduced in 2000, for instance, has only a very limited response in 2000 itself but causes a 30\% reduction of global carbon emissions after 10 years - and reduces global emissions by more than $50 \%$ after 30 years. 'Baseline learning', 'induced learning' and 'inertia' all contribute to this increasing response in time.

In a second experiment we bring in the time of introduction of the tax $\left(t_{\text {in }}\right)$. What happens if the tax is not introduced in 2000, but in 2010 or 2020? We recorded the impact in $2030\left(t_{\text {rec }}\right)$ of three different series of taxes introduced in 2000, 2010 and 2020, respectively (Fig. 8b). The results are fairly similar to the previous experiment. A tax introduced in 2000 has the largest response, benefiting again from both baseline and induced learning processes, and having sufficient time to overcome the existing inertia. The 2030 response to a tax introduced in 2020 is significantly smaller. Interestingly, this 

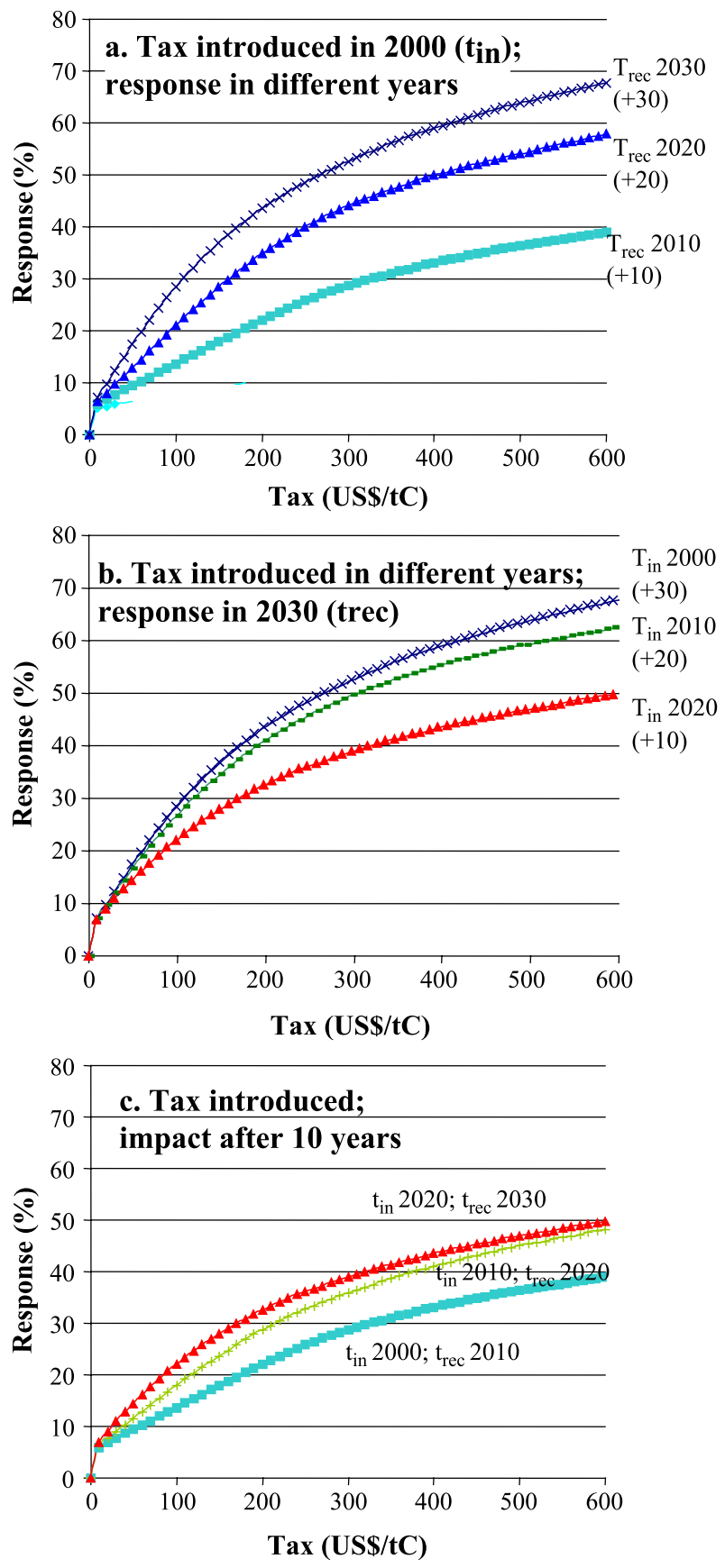

Fig. 8. Response to a carbon tax, (a) introduction year 2000; different recording years. (b) Recording year 2030; different introduction years. (c) Recording year 10 years after introduction year. 
curve lies some 10\% above the curve in Fig. 8a of the 2010 response of tax introduced in 2000 (both curves are included in Fig. 8c). In terms of time elapsed after the tax was introduced these cases are similar as both curves show the situation 10 years after the tax was introduced. Assuming that the role of inertia and induced learning will therefore be comparable, in particular technology development under the baseline can be identified as an important process explaining these differences. Fig. 8c shows all three curves, recorded 10 years after the introduction of the tax.

We continue this line of thinking by now considering the introduction time $t_{\text {in }}$ and the recording time $t_{\text {rec }}$ as two independent axes on one graph. In this graph we show for a given tax level $T$ (in this case US\$300/tC) all possible responses as a function of combinations of $t_{\text {in }}$ and $t_{\text {rec }}$, in 5-year steps. The surface that is created in this way obviously shows the strongest response in the lower right corner, as this depicts the situation of an early introduction of the tax (2000) and late recordings (2030). The diagonal from the left low corner $\left(t_{\text {in }}=2000, t_{\text {rec }}=2000\right)$ to the right upper corner $\left(t_{\text {in }}=2030 ; t_{\text {rec }}=2030\right)$ represents all points in which response is recorded immediately after the introduction of the tax - and responses along this diagonal are therefore small. All points going to the left upper corner from this diagonal are zero by definition (recording time before the introduction of the tax). This representation allows for a comparison in different directions. Horizontal and vertical lines through the graph show the influence of changes in recording time and introduction time, respectively, while diagonals compare cases with a constant time between $t_{\text {in }}$ and $t_{\text {rec }}$. The highlighted diagonal in the graph, for instance, shows all cases with a 20 -year time period between introduction of the tax and recordings for the 2020-2030 period.

We will first look at the results of this graph in the normal model mode (Fig. 9; left upper graph). A US $\$ 300 / \mathrm{tC}$ tax gives a maximum response of almost $60 \%$ reduction of global $\mathrm{CO}_{2}$ emissions if introduced in 2000 and recorded in 2030 (right low corner). An important observation is that the graph is not symmetrical in its response to the two different time axes. The cause of this is mainly the 'learning under the baseline' that creates different starting situations for our experiments.

In the model, we can now step-by-step switch off different dynamics. First, the additional 'learning-by-doing' induced by a carbon tax is completely switched off (learning is equal to baseline), resulting in Fig. 9 (upper right graph). Instead of reaching a maximum reduction near $60 \%$ in the maximum reduction is now $40-50 \%$ (right low corner). Thus, induced learning between 2000 and 2030 to a US\$300/tC tax creates an additional $10 \%$ response under the $\mathrm{B} 2$ assumptions to the response that would be obtained if no induced learning was included in the model. Interestingly, the difference between the first and second graph becomes less for the cases with a shorter period between the year of introduction and the recording time. This result can be understood, as this also decreases the period in which induced learning can take place.

In the last experiment, we also switched off all learning that had already occurred in the baseline-leaving all technology frozen at its 2000 level $^{7}$ (Fig. 9; lower left). This means that now mainly system inertia determine our results. Again, taking out the process of

\footnotetext{
7 Obviously, this also changes the baseline itself in terms of emissions. However, as we are interested in relative responses, this does not create major obstacles for comparing the different cases.
} 

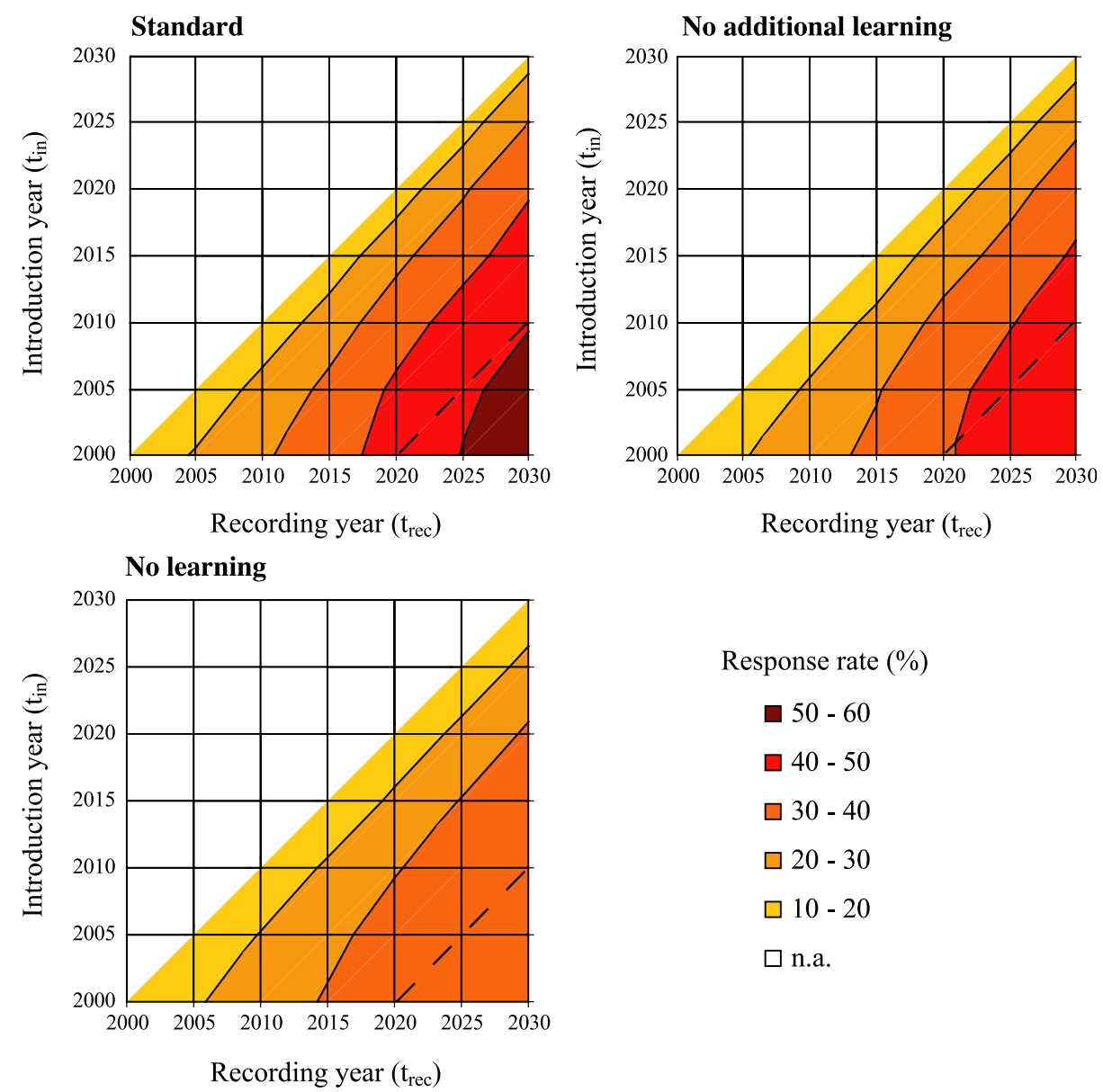

Fig. 9. Global carbon response rate (in \% reduction compared to baseline) to a US\$300/tC tax as a function of introduction and recording year. The introduction year represent the year the tax is introduced, the recording year the year that the response to the tax is recorded. The dashed line indicates, as example, all points in which the response is recorded 20 years after the introduction.

technological development has reduced the response of the system to the carbon tax. The maximum response is now around 35\% for the 2000 introduction, and 2030 as recording year (right low corner), thus again a loss of about $10 \%$ in terms of carbon emission reduction. A second observation is that the graph has become more symmetrical. This complies to our explanation that the asymmetric response in first two graphs of Fig. 9 is at least partly related to learning under the baseline. The remaining asymmetry is caused largely by depletion of fossil fuels in time (weakening the competitive position of fossil-fuel based technologies).

This set of experiments shows the importance of (assumptions about) technology development for the effectiveness of reducing carbon dioxide emissions - and for the abatement costs, if we take the level of the carbon tax as a proxy of costs. In our 
experiments, we have more-or-less untangled the different roles of technology development in the baseline, induced technology development and inertia. Both induced technology development and technology development in the baseline contributed to $10 \%$ more reduction of carbon dioxide emissions in case of a US\$300 tax introduced in 2000 and recorded in 2030. Inertia is very important as well, and on its own it causes a difference between a $10 \%$ reduction of global emissions after 5 years and a $35 \%$ reduction after 30 years. It should be noted that these results reflect the full dynamics in the (simulated) world energy system, including depletion and trade.

\section{Discussion and main conclusions}

We have studied a set of different mitigation experiments, with a particular focus on the role of technologies in terms of mitigation response to a carbon tax.

In interpreting the results of these experiments, we obviously need to realize the model characteristics and assumptions. TIMER is an energy system model with a strong focus on relevant dynamic relationships among the various mitigation options but without macroeconomic feedbacks. A second point refers to the baseline and the options that were used in our mitigation scenarios. The IMAGE B2 baseline, used as a baseline for our analysis, should be regarded as a medium- to low-emission scenario. As a result, most of the reductions that have been studied here can be regarded as reductions with a medium level of ambition (e.g. a $40 \%$ reduction of carbon emissions by 2100 is required to reach stabilization at $550 \mathrm{ppmv}$ ). On the other hand, the TIMER 1.0 version does not include all available mitigation options, in particular, carbon sequestration, neither by means of capture and storage or by means of sinks enhancement.

Using an energy-model in the context of an integrated assessment model allows us to study some indirect changes of climate policies as well. First of all, the changes in the energy system in response to the carbon tax not only change carbon emissions but also other greenhouse gases and sulfur emissions. We have shown in this paper that the environmental effectiveness - certainly in the short term-is limited as a result of a reduction in the aerosol cooling effect. Secondly, using integrated analysis can show some of the trade-offs between reducing energy-related carbon dioxide emission by using biofuels and its impact on land-use emissions. In our current results, biofuel use has a net mitigation effect, but some of the mitigation is offset by the additional demand for agricultural land, which increases land-use emissions.

The results, above all, indicate how important technological improvement is for climate mitigation strategies. This is shown, for instance, by cost reductions in solar/wind technology in the case of the first two experiments. This is shown the clearest with the results of the last set of experiments. Leaving out all forms of technology development reduces the response to a US $\$ 300 / \mathrm{tC}$ carbon tax in 2030 from a $60 \%$ reduction to only $30 \%$ (both compared to baseline). Partly as a result of these technology developments, stabilization of 550 ppmv from the IMAGE B2 baseline appears feasible at relatively low costs by introducing a uniform carbon tax and the variety of measures induced by this tax. Interestingly, the costs and measures taken in going from B2 to 550 stabilization are moreor-less comparable to those found earlier for going to $450 \mathrm{ppmv}$ stabilization from the B1 
baseline (van Vuuren and de Vries, 2001). This shows how important baseline assumptions can be for the costs of reaching different stabilization levels; in particular, the sustainable development orientation and the strong technology development assumed in the B1 baseline can allow for reaching lower stabilization levels at bearable costs when compared to other baseline scenarios.

Breaking down the results for the B2-550 stabilization scenario shows improved efficiency to be the single most important factor in the first decades in terms of the mitigation response, followed by fuel-switching among the fossil fuels (in particular from coal to natural gas). However, from 2030 onwards, introduction of carbon-free supply options provides the bulk of the required reductions. As a result, the changes in global energy intensity remain near the upper end of the historically observed range, whereas decarbonization rates go the levels above historical rates for the whole century. In terms of energy carriers the sharpest reductions take place for coal: a 50\% reduction in cumulative coal use. This implies that the greatest changes take place in regions with high shares of coal consumption or production. Alternatively, these regions might need to develop carbon storage (excluded in our experiments). In terms of fuel trade, carbon-tax induced oil trade changes appear to be modest. Changes in trade of other energy carriers may be of the same order of magnitude and, depending on the region, work in the same direction as changes in oil trade or completely offset them. The latter is, for instance, the case for the Former Soviet Union, where one could see how natural gas and biofuels exports offset the losses in oil exports.

Technology development needs to be studied in the context of other dynamic processes important in the world energy system. In our simulated B2 world of the TIMER model, early-action type of scenarios results in accelerated technology development on the short and medium term. In the long run, however, there are a number of processes that may work in the opposite direction, such as the maximum share of renewable technologies that can be absorbed in the electric power system without additional costs, and the impacts on depletion of both fossil fuels and renewables. The exact results depend on the different assumptions made. In the current runs, scenarios with early carbon taxes still have lower carbon dioxide concentrations in 2100; however, these scenarios show a similar emission reduction in 2100 as the scenarios with a slower introduction of the same carbon tax. It is important to study the role of these processes in more detail.

Three technological processes that have a direct influence on the mitigation response to carbon taxes are the technology development in the baseline, induced technology development as a result of climate policy and inertia. The relative importance of these different processes is directly related to the discussion on timing of mitigation action. In our analyses, we have indicated how these processes all play a role. Learning that is part of the baseline indeed makes a 2030 response to a 2020 tax, 5-10\% larger than a 2010 response to a 2010 tax. However, the other two processes that work in the opposite direction are at least as strong. Induced learning results in $10 \%$ more reduction to a US $\$ 300 / \mathrm{tC}$ tax in 2030; and without learning, inertia results in a difference a $10 \%$ reduction of global emissions after 5 years and a 35\% reduction after 30 years. Taken together, in this very short time period of evaluation, the processes that would support an early action response seem to dominate over the processes that favor a delayed response 
approach - at least, if no discount rate is applied. In any case, the dynamics behind different technological process have been found to be very important. Providing sufficient pressure to stimulate technology development in the direction of low-carbon energy systems seems to be crucial. Sufficient resources for research and development and climate policies can help the developments in this direction.

\section{References}

Alcamo, J., Leemans, R., Kreileman, E. (Eds.), 1998. Global Change Scenarios of the 21st Century. Results from the Image 2.1 Model. Pergamon \& Elsevier Science, London. 286 pp.

Argotte, L., Epple, D., 1990. Learning curves in manufacturing. Science 247, 920-924.

Azar, C., Dowlatabadi, H., 1999. A review of technical change in assessment of climate policy. Annual Review of Energy and the Environment 24, 513-544.

Criqui, P., Mima, S., Viguier, L., 1999. Marginal abatement costs of $\mathrm{CO}_{2}$ emission reductions, geographical flexibility and concrete ceilings: an assessment using the POLES model. Energy Policy 27 (10), 585-601.

De Vries, H.J.M., Van Vuuren, D.P., Den Elzen, M.G.J., Janssen, M.A., 2001. The Targets Image Energy Regional (TIMER) Model. Technical Documentation. RIVM Report no. 461502024. National Institute of Public Health and the Environment, Bilthoven, The Netherlands.

Edmonds, J., Reilly, J., 1985. Global Energy-Assessing the Future. Oxford Univ. Press, New York. 400 pp.

Ellerman, A.D., Decaux, A., 1998. Analysis of Post-Kyoto $\mathrm{CO}_{2}$ emissions trading using marginal abatement curves. Report No 40, MIT, Cambridge, MA.

Grubb, M., 1997. Technologies, energy systems and the timing of $\mathrm{CO}_{2}$ emissions abatement-an overview of economic issues. Energy Policy 25 (2), 159-172.

Grubler, A., Nakicenovic, N., Victor, D.G., 1999. Dynamics of energy technologies and global change. Energy Policy 27 (5), 247-280.

Ha-Duong, M., Grubb, M.J., Hourcade, J.C., 1997. Influence of socioeconomic inertia and uncertainty on optimal $\mathrm{CO}_{2}$-emission abatement. Nature 390 (6657), 270-273.

Hoogwijk, M., Broek, R.v.d., Berndes, G., Faaij, A., 2000. A review of assessments on the future global contribution of biomass energy. 1st World Conference and Exhibition on Biomass for Energy and Industry, Sevilla.

IMAGE Team, 2001. The IMAGE 2.2 Implementation of the SRES Scenarios. A comprehensive analysis of emissions, climate change and impacts in the 21st century. RIVM CD-ROM publication 481508018, National Institute for Public Health and the Environment, Bilthoven, The Netherlands.

IPCC, 2001a. Climate Change 2001: Mitigation. Cambridge Univ. Press, Cambridge.

IPCC, 2001b. Climate Change 2001: Synthesis Report. Cambridge Univ. Press, Cambridge, UK. 398 pp.

Nakicenovic, A., et al., 2000. Special Report on Emissions Scenarios (SRES). Cambridge Univ. Press, Cambridge.

Riahi, K., Roehrl, R.A., 2000. Greenhouse gas emissions in a dynamics-as-usual scenario of economic and energy development. Technological Forecasting \& Social Change 63 (2-3).

Rogner, H.H., 1997. An Assessment of world hydrocarbon resources. Annual Review of Energy and the Environment 22, 217-262.

van Vuuren, D.P., de Vries, H.J.M., 2001. Mitigation scenarios in a world oriented at sustainable development: the role of technology, efficiency and timing. Climate Policy 1, 189-210.

van Vuuren, D., Fengxi, Z., De Vries, B., Kejun, J., Graveland, C., Yun, L., 2003. Energy and emission scenarios for China in the 21st century-exploration of baseline development and mitigation options. Energy Policy 31, $369-387$.

WEA, 2000. World Energy Assessment—Energy and the Challenge of Sustainability. United Nations Development Programme, New York.

Wene, C.O., 2000. Experience Curves for Energy Technology Policy. OECD/IEA, Paris.

Wigley, T.M.L., Richels, R., Edmonds, J.A., 1996. Economic and environmental choices in stabilisation of atmospheric $\mathrm{CO}_{2}$ concentrations. Nature 369, 240-243. 